GLASNIK MATEMATIČKI

Vol. 55(75)(2020), $67-83$

\title{
ON THE FAITHFULNESS OF 1-DIMENSIONAL TOPOLOGICAL QUANTUM FIELD THEORIES
}

\author{
Sonja Telebaković Onić \\ University of Belgrade, Serbia
}

\begin{abstract}
This paper explores 1-dimensional topological quantum field theories. We separately deal with strict and strong 1-dimensional topological quantum field theories. The strict one is regarded as a symmetric monoidal functor between the category of 1-cobordisms and the category of matrices, and the strong one is a symmetric monoidal functor between the category of 1-cobordisms and the category of finite dimensional vector spaces. It has been proved that both strict and strong 1-dimensional topological quantum field theories are faithful.
\end{abstract}

\section{INTRODUCTION}

The concept of topological quantum field theory goes back to the work of Witten ([19]). Mathematical axioms for topological quantum field theories are given by Atiyah ([1]). The categorical viewpoint is developed in Quinn's lectures ([16]). According to Atiyah and Quinn, an n-dimensional topological quantum field theory $(n-\mathrm{TQFT})$ is a symmetric monoidal functor from the category of oriented $n$-cobordisms to the category of finite dimensional vector spaces over some field. Such functor associates a vector space with each closed oriented $(n-1)$-dimensional manifold and associates a linear map with each oriented $n$-cobordism. It is well known that there is a one-to-one correspondence between 2-TQFTs and commutative Frobenius algebras (the best reference here is [12, Theorem 3.3.2]). For another proof of this classical correspondence see [11, Theorem 4.1].

2020 Mathematics Subject Classification. 15A69, 15B34, 18M05, 57R56.

Key words and phrases. Symmetric monoidal category, oriented manifold, cobordism, topological quantum field theory, Brauerian representation, Kronecker product, commutation matrix. 
The faithfulness property of TQFTs is very important, since it provides a complete set of invariants for the classification of cobordisms. However, the faithfulness problem of TQFTs is seldom investigated in relevant literature. Inspired by the representation of a class of diagrammatic algebras given by Brauer ([3]), Došen and Petrić have obtained a result that demonstrates the faithfulness of a 1-TQFT (see [7, Section 14]). This example of a faithful 1-TQFT maps the 0-dimensional sphere $S^{0}$ to a matrix Frobenius algebra, as explained in [2, Section 5]. It is shown by Gajović, Petrić and the author ([9]) that there is a faithful 2-TQFT which corresponds to the commutative Frobenius algebra $\mathbb{Q} \mathbb{Z}_{5} \otimes Z\left(\mathbb{Q S}_{3}\right)$, the tensor product of the group algebra and the center of the group algebra. One question remains unanswered: is there a faithful $n$-TQFT, for $n \geq 3$ ?

The aim of this paper is to prove the faithfulness of all 1-TQFTs, mapping the 0-dimensional manifold consisting of one point to a vector space of dimension at least 2. In Section 2, we summarize the relevant material on the category of 1-cobordisms, and associate a $0-1$ matrix with each 1cobordism. This association is motivated by Brauer's matrix representation of the class of diagrammatic algebras ([3]). A generalization of Brauer's representation given by Došen and Petrić $([6,7])$ leads to a symmetric monoidal functor between the category of 1-cobordisms and the category of matrices. In this way we obtain what will be referred to as the Brauerian functor. Our results on the faithfulness of this functor are presented in Section 3. In Section 4, we introduce the notion of a strict 1-TQFT in order to use matrix techniques. The main result of this section is that every strict 1-TQFT, $F: 1 C o b \rightarrow M a t_{\mathbb{K}}$, mapping the 0 -dimensional manifold consisting of one point to a natural number $p \geq 2$, is faithful, since it coincides with the Brauerian functor up to multiplication by invertible matrices. Roughly speaking, the faithfulness of $F$ means that $F(K)$ is a complete set of algebraic invariants for 1-cobordisms. The last section is devoted to the study of strong 1-TQFTs (symmetric strong monoidal functors between the category of 1-cobordisms and the category of finite dimensional vector spaces over a chosen field). We extend our faithfulness result to the case of strong 1-TQFTs.

Throughout the paper by a 1-dimensional manifold we mean a compact, oriented 1-dimensional manifold with boundary.

\section{The Category $1 C o b$}

Objects of the category $1 \mathrm{Cob}$ are closed oriented 0-dimensional manifolds, consisting of a finite number of points. Every particular point is associated with a sign, that represents its orientation. From now on, we will consider the objects of $1 C o b$ as ordered pairs $(n, \varepsilon)$, where $n=\{0, \ldots, n-1\}$, and $\varepsilon: n \rightarrow\{-1,1\}$. 
The morphisms of $1 \mathrm{Cob}$ are the equivalence classes of 1-cobordisms $\left(M, f_{0}:\left(n, \varepsilon_{0}\right) \rightarrow M, f_{1}:\left(m, \varepsilon_{1}\right) \rightarrow M\right)$, where $M$ is a compact oriented 1-dimensional manifold, such that its boundary $\partial M$ is a disjoint union of $\Sigma_{0}$, and $\Sigma_{1} ; f_{0}$ is an orientation preserving embedding which image is $\Sigma_{0}$, while $f_{1}$ is an orientation reversing embedding which image is $\Sigma_{1}$. The manifolds $\Sigma_{0}$ and $\Sigma_{1}$ are called the ingoing and outgoing boundary of $M$, respectively. Two 1-cobordisms $K=\left(M, f_{0}, f_{1}\right)$ and $K^{\prime}=\left(M^{\prime}, f_{0}^{\prime}, f_{1}^{\prime}\right)$ are equivalent, denoted by $K \sim K^{\prime}$, if there is an orientation preserving homeomorphism $F: M \rightarrow M^{\prime}$ such that the following diagram commutes.

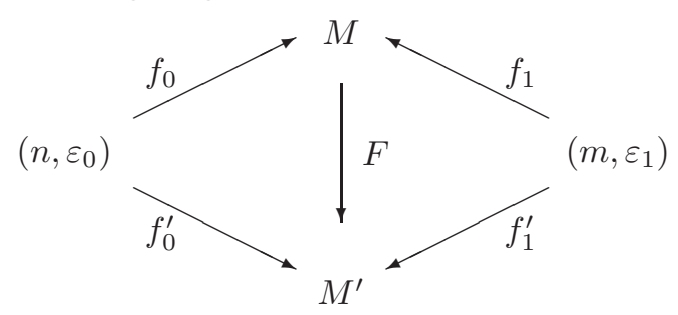

The category $1 C o b$ is a strict monoidal with respect to the sum on objects

$$
\left(n, \varepsilon_{0}\right)+\left(n^{\prime}, \varepsilon_{1}\right)=\left(n+n^{\prime}, \varepsilon_{0}+\varepsilon_{1}\right),
$$

where $\varepsilon_{0}+\varepsilon_{1}: n+n^{\prime} \rightarrow\{-1,1\}$ is defined by

$$
\left(\varepsilon_{0}+\varepsilon_{1}\right)(x)= \begin{cases}\varepsilon_{0}(x), & \text { if } x \in n, \\ \varepsilon_{1}(x-n), & \text { if } x \notin n,\end{cases}
$$

and the operation of "putting side by side" on morphisms, denoted by $\otimes$.

The category $1 C o b$ is also a symmetric monoidal with respect to the family of cobordisms $\tau_{n, m}:\left(n+m, \varepsilon_{0}+\varepsilon_{1}\right) \rightarrow\left(m+n, \varepsilon_{1}+\varepsilon_{0}\right)$ corresponding to permutations on $n+m$. For example, $\tau_{3,2}$ is illustrated by the following picture

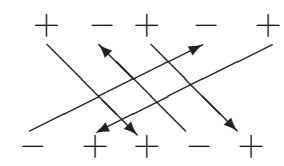

Every morphism of $1 \mathrm{Cob}$ is completely determined by a 1 -dimensional manifold $M$ and two sequences of its boundary points, denoted by

$$
\begin{aligned}
& (0,0),(1,0), \ldots,(n-1,0), \\
& (0,1),(1,1), \ldots,(m-1,1),
\end{aligned}
$$

where the first one corresponds to the ingoing boundary $\Sigma_{0}$, and the second one to the outgoing boundary $\Sigma_{1}$.

Every 1-cobordism $K=\left(M, \Sigma_{0}, \Sigma_{1}\right):\left(n, \varepsilon_{0}\right) \rightarrow\left(m, \varepsilon_{1}\right)$ induces the following equivalence relation $R_{K}$ on the set $(n \times\{0\}) \cup(m \times\{1\})$. For $(i, k),(j, l)$ 
elements of $(n \times\{0\}) \cup(m \times\{1\})$, we have that

$$
((i, k),(j, l)) \in R_{K}
$$

$\Leftrightarrow$

points $(i, k)$ and $(j, l)$ belong to the same connected component of $M$.

Let $c_{K}$ denote the number of connected components of $K$, which are homeomorphic to the circle $S^{1}$. It is clear that for cobordisms $K, L:\left(n, \varepsilon_{0}\right) \rightarrow$ $\left(m, \varepsilon_{1}\right)$ the following proposition holds.

Proposition 2.1. The 1-cobordisms $K$ and $L$ are equivalent iff $R_{K}=R_{L}$ and $c_{K}=c_{L}$.

Let $X$ be an arbitrary set, let $R \subseteq X^{2}$ be an equivalence relation on $X$ and let $p$ be the set $\{0,1, \ldots, p-1\}, p \geq 2$. Consider the following set of functions:

$$
\mathcal{F}^{=}(R)=\{f: X \rightarrow p \mid(\forall x, y \in X)((x, y) \in R \Rightarrow f(x)=f(y))\} .
$$

Proposition 2.2. (Došen and Petrić [4], [5]) If $R_{1}, R_{2} \subseteq X^{2}$ are equivalence relations, then $R_{1}=R_{2}$ iff $\mathcal{F}=\left(R_{1}\right)=\mathcal{F}=\left(R_{2}\right)$.

We associate a non-zero matrix $A(K)$ of order $p^{m} \times p^{n}$ with each cobor$\operatorname{dism} K:\left(n, \varepsilon_{0}\right) \rightarrow\left(m, \varepsilon_{1}\right)$ in the following way. Let $R_{K} \subseteq((n \times\{0\}) \cup$ $(m \times\{1\}))^{2}$ be equivalence relation corresponding to the cobordism $K$. The number of rows of $A(K)$ is equal to the number of functions $m \rightarrow p$. Each of these functions can be envisaged as a sequence of length $m$ of elements of $\{0,1, \ldots, p-1\}$. The set of these sequences may be ordered lexicographically so that $00 \ldots 0$ is the first, and $(p-1)(p-1) \ldots(p-1)$ is the last in this ordering. Since $\left(p^{m}, \leq\right)$ is isomorphic to the set of these sequences, the rows of $A(K)$ can be identified by functions from $m$ to $p$. Let $f_{i}: m \rightarrow p$ denote the function corresponding to the $i$-th row. Columns of $A(K)$ can be identified by functions from $n$ to $p$. Let $g_{j}: n \rightarrow p$ denote the function corresponding to the $j$-th column. Let $\left[g_{j}, f_{i}\right]:(n \times\{0\}) \cup(m \times\{1\}) \rightarrow p$ be the function defined by

$$
\left[g_{j}, f_{i}\right](x)= \begin{cases}g_{j}\left(\pi_{0}(x)\right), & \text { if } x \in n \times\{0\} \\ f_{i}\left(\pi_{1}(x)\right), & \text { if } x \in m \times\{1\}\end{cases}
$$

where $\pi_{0}: n \times\{0\} \rightarrow n$ and $\pi_{1}: m \times\{1\} \rightarrow m$ are bijections given by $\pi_{0}(u, 0)=u$ and $\pi_{1}(v, 1)=v$, respectively. Element $A(K)[i, j]$ in the $i$-th row and $j$-th column of $A(K)$ is equal to 1 iff $\left[g_{j}, f_{i}\right] \in \mathcal{F}=\left(R_{K}\right)$, otherwise it is 0 .

EXAMPLE 2.3. If $K:\left(4, \varepsilon_{0}\right) \rightarrow\left(2, \varepsilon_{1}\right)$ is 1 -cobordism illustrated by the following picture

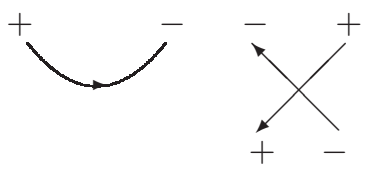


and $p=2$, the corresponding matrix $A(K)$ is equal to

\begin{tabular}{|c|c|c|c|c|c|c|c|c|c|c|c|c|c|c|c|}
\hline 0000 & 0001 & 0010 & 0011 & 0100 & 0101 & 0110 & 0111 & 1000 & 1001 & 1010 & 1011 & 1100 & 1101 & 1110 & 1111 \\
\hline 00 & 0 & 0 & 0 & 0 & 0 & 0 & 0 & 0 & 0 & 0 & 0 & 1 & 0 & 0 & 0 \\
\hline 0 & 0 & 1 & 0 & 0 & 0 & 0 & 0 & 0 & 0 & 0 & 0 & 0 & 0 & 1 & 0 \\
\hline 0 & 1 & 0 & 0 & 0 & 0 & 0 & 0 & 0 & 0 & 0 & 0 & 0 & 1 & 0 & 0 \\
\hline 0 & 0 & 0 & 1 & 0 & 0 & 0 & 0 & 0 & 0 & 0 & 0 & 0 & 0 & 0 & 1 \\
\hline
\end{tabular}

For example, element $A(K)[2,3]$ is equal to 1 , since the sequences 01 and 0010 corresponding to the second row and the third column "match" into the picture.

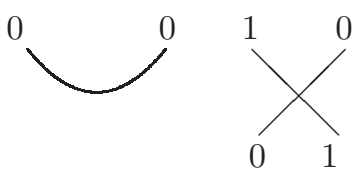

\section{Brauerian REPRESENTATION}

Let $M a t_{\mathbb{K}}$ be a category whose objects are natural numbers, and whose morphisms from $n$ to $m$ are all $m \times n$ matrices over the field $\mathbb{K}$ of characteristic zero. The composition of morphisms is matrix multiplication, and the identity morphism $n \rightarrow n$ is the identity matrix of order $n$. The category $\left(M_{\mathbb{K}}, \otimes, 1, S_{n, m}\right)$ is a symmetric strict monoidal with respect to the multiplication on objects, and the Kronecker product on morphisms, and the family of $m n \times n m$ matrices $S_{n, m}$. The matrix $S_{n, m}$ is the matrix representation of the linear map $\sigma: \mathbb{K}^{n} \otimes \mathbb{K}^{m} \rightarrow \mathbb{K}^{m} \otimes \mathbb{K}^{n}$ with respect to the standard ordered bases, defined on the basis vectors by $\sigma\left(e_{i} \otimes f_{j}\right)=f_{j} \otimes e_{i}$. We call $S_{n, m}$ the commutation matrix.

Let us now consider the following functor $B$ from $1 C o b$ to $M a t_{\mathbb{K}}$. It is defined by $B(n, \varepsilon)=p^{n}$ on objects, where $p \geq 2$. For morphism $K:\left(n, \varepsilon_{0}\right) \rightarrow$ $\left(m, \varepsilon_{1}\right)$ of $1 C o b$, it is given by $B(K)=p^{a} \cdot A(K)$, where $a$ is the number of circular components of $K$, and $A(K)$ is $(0,1)$-matrix of order $p^{m} \times p^{n}$ associated with $K$.

It can be proved that the following proposition holds.

Proposition 3.1. B is a strict symmetric monoidal functor from $\left(1 C o b, \otimes,(0, \varepsilon), \tau_{n, m}\right)$ to $\left(M_{a t} t_{\mathbb{K}}, \otimes, 1, S_{n, m}\right)$.

The proof of this proposition can be adapted from [5, Section 5, Proposition 4].

The functor $B$ is related to the matrix representation of a class of diagrammatic algebras given by Brauer (see [3],[6] for more details). Therefore, we call $B$ Brauerian functor.

Proposition 3.2. B is faithful. 
Proof. Suppose that $K, L:\left(n, \varepsilon_{0}\right) \rightarrow\left(m, \varepsilon_{1}\right)$ are two morphisms of $1 C o b$ such that $B(K)=B(L)$. Let $a$ and $b$ denote numbers of circular components of $K$ and $L$, respectively. Since the non-zero matrices $p^{a} \cdot A(K)$ and $p^{b} \cdot A(L)$ are equal, they have the same order $p^{m} \times p^{n}$ and the corresponding elements, which are either zero or the powers of $p$, are identical. We have $a=b$, because $p \geq 2$, and

$$
A(K)[i, j]=1 \text { iff } A(L)[i, j]=1 \text {. }
$$

This means that for every $g_{j}: n \rightarrow p$ and every $f_{i}: m \rightarrow p$ we have

$$
\left[g_{j}, f_{i}\right] \in \mathcal{F}^{=}\left(R_{K}\right) \text { iff }\left[g_{j}, f_{i}\right] \in \mathcal{F}=\left(R_{L}\right) .
$$

As every function $f:(n \times\{0\}) \cup(m \times\{1\}) \rightarrow p$ is of the form $\left[g_{j}, f_{i}\right]$ for some $g_{j}: n \rightarrow p$ and some $f_{i}: m \rightarrow p$, it follows that $\mathcal{F}=\left(R_{K}\right)=\mathcal{F}=\left(R_{L}\right)$. Applying Proposition 2.2, we conclude that $R_{K}=R_{L}$. By Proposition 2.1 it follows that $K$ and $L$ are equivalent.

It is worth pointing out that the faithfulness of the Brauerian functor $B$ is a consequence of the maximality given in [7, Section 14].

\section{Strict 1-Dimensional Topological Quantum Field Theories}

Let us denote by + a 0 -dimensional manifold, which consists of one point with positive orientation, and by - the same manifold with the opposite orientation. Then, every object of $1 C o b$ can be regarded as a sequence $a_{1} \ldots a_{n}$, where $a_{i} \in\{+,-\}$. Any 1-dimensional manifold $M$ is homeomorphic to a disjoint union of its connected components. Those connected components are either homeomorphic to a closed interval $[0,1]$ or to a circle $S^{1}$. Depending on how the boundary $\partial M$ is decomposed into ingoing and outgoing pieces, we have the following connected cobordisms:

$\mathbf{1}^{\mathbf{0}}$ Unit interval $[0,1]$ with its standard orientation regarded as a cobordism from + to + . It represents the identity morphism $i d_{+}$in 1 Cob.

$2^{\mathbf{0}}$ Unit interval $[0,1]$ with its standard orientation regarded as a cobordism from - to - . It represents the identity morphism $i d_{-}$in $1 C o b$.

$\mathbf{3}^{\mathbf{0}}$ Unit interval $[0,1]$ with its standard orientation regarded as a cobordism from +- to $\emptyset$. It represents the morphism of $1 C o b$, which we denote by $\mathcal{B}$.

$4^{0}$ The cobordism from -+ to $\emptyset$, which we denote by $\overline{\mathcal{B}}$.

$5^{0}$ Unit interval $[0,1]$ with its standard orientation regarded as a cobordism from $\emptyset$ to -+ . It represents the morphism of $1 C o b$, which we denote by $\mathcal{C}$.

$\mathbf{6}^{\mathbf{0}}$ The cobordism from $\emptyset$ to +- , which we denote by $\overline{\mathcal{C}}$.

$7^{0}$ The circle $S^{1}$ regarded as a cobordism from the empty set to itself. 


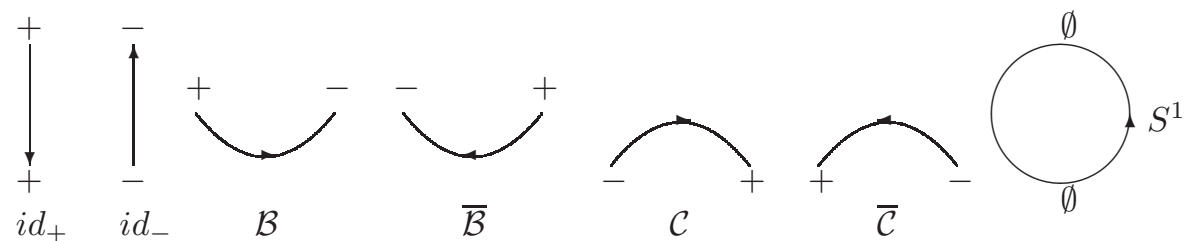

Definition 4.1. A strict 1-dimensional topological quantum field theory (1-TQFT) is a strict symmetric monoidal functor between the category $1 C o b$ and the category $M a t_{\mathbb{K}}$.

The following proposition is motivated by [13, Proposition 1.1.8].

Proposition 4.2. Let $F$ be a strict 1-TQFT. If $p=F(+)$ and $q=F(-)$, then $p=q$.

Proof. Applying the functor $F$ to the morphism $\mathcal{B}:+-\rightarrow \emptyset$ of $1 C o b$, we obtain the morphism $F(\mathcal{B}): F(+-) \rightarrow F(\emptyset)$ of $M a t_{\mathbb{K}}$. Since the functor $F$ is a strict monoidal, we have $F(+-)=F(+) \otimes F(-)=p \cdot q$ and $F(\emptyset)=1$. Hence, the matrix $F(\mathcal{B})$ has order $1 \times(p q)$. Let us introduce the notation

$$
F(\mathcal{B})=\left[\begin{array}{lllllll|l|lll}
\beta_{11} & \ldots & \beta_{1 q} & \beta_{21} & \ldots & \beta_{2 q} & \ldots & \beta_{p 1} & \ldots & \beta_{p q}
\end{array}\right]
$$

and

$$
X=\left[\begin{array}{ccc}
\beta_{11} & \ldots & \beta_{1 q} \\
\beta_{21} & \ldots & \beta_{2 q} \\
\vdots & \vdots & \vdots \\
\beta_{p 1} & \ldots & \beta_{p q}
\end{array}\right]
$$

Under the standard isomorphism $H: \mathcal{M}_{p \times q} \rightarrow \mathcal{M}_{1 \times p q}$, we have $H(X)=$ $F(\mathcal{B})$.

Similarly, applying the functor $F$ to the morphism $\mathcal{C}: \emptyset \rightarrow-+$, we obtain the morphism $F(\mathcal{C}): 1 \rightarrow q \cdot p$ of $M_{a t}$, i.e. the matrix of order $(q p) \times 1$. Set

$$
F(\mathcal{C})=\left[\begin{array}{lllllllll|lll}
\gamma_{11} & \ldots & \gamma_{1 p} & \mid & \gamma_{21} & \ldots & \gamma_{2 p} & \ldots & \gamma_{q 1} & \ldots & \gamma_{q p}
\end{array}\right]^{T}
$$

and

$$
Y=\left[\begin{array}{ccc}
\gamma_{11} & \ldots & \gamma_{1 p} \\
\gamma_{21} & \ldots & \gamma_{2 p} \\
\vdots & \vdots & \vdots \\
\gamma_{q 1} & \ldots & \gamma_{q p}
\end{array}\right] .
$$

Under the standard isomorphism $L: \mathcal{M}_{q \times p} \rightarrow \mathcal{M}_{q p \times 1}$, we have $L(Y)=F(\mathcal{C})$.

We consider the identity cobordism $i d_{+}$and its decomposition into two cobordisms $i d_{+} \otimes \mathcal{C}$ and $\mathcal{B} \otimes i d_{+}$, as shown in the picture below. 


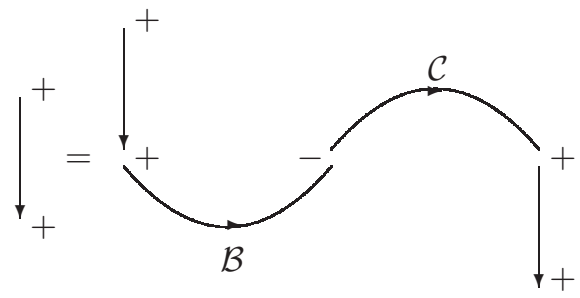

Applying the functor $F$ to both sides of the equation $i d_{+}=\left(\mathcal{B} \otimes i d_{+}\right) \circ\left(i d_{+} \otimes \mathcal{C}\right)$ and using the functorial properties of $F$, we obtain

$$
i d_{F(+)}=\left(F(\mathcal{B}) \otimes i d_{F(+)}\right) \cdot\left(i d_{F(+)} \otimes F(\mathcal{C})\right),
$$

i.e.

$$
E_{p}=\left(F(\mathcal{B}) \otimes E_{p}\right) \cdot\left(E_{p} \otimes F(\mathcal{C})\right),
$$

where $E_{p}$ is the identity matrix of order $p$. As a result of matrix multiplication, we get the system of $p^{2}$ equations

$$
\sum_{k=1}^{q} \beta_{i k} \cdot \gamma_{k j}=\delta_{i j}, \quad i, j \in\{1, \ldots, p\},
$$

that can be written in the matrix form

$$
X \cdot Y=E_{p} .
$$

Therefore, the matrix $X \in \mathcal{M}_{p \times q}$ has a right inverse, so its rows are linearly independent, i.e. row rank is equal to $p$.

If we apply the functor $F$ to the morphism $\tau_{-+}:-+\rightarrow+-$ of $1 \mathrm{Cob}$, shown in the following picture

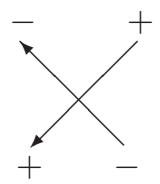

we obtain the morphism $F\left(\tau_{-+}\right): F(-) \otimes F(+) \rightarrow F(+) \otimes F(-)$ of $M a t_{\mathbb{K}}$, i.e. the matrix of order $(p q) \times(q p)$. The symmetry of $F$ implies $F\left(\tau_{-+}\right)=$ $S_{q, p}$, where $S_{q, p}$ is the commutation matrix. The crucial fact is that the commutative matrix $S_{q, p}$ satisfies the following conditions:

$$
\begin{gathered}
S_{q p} \cdot L(Y)=L\left(Y^{T}\right), \\
H(X) \cdot S_{q p}=H\left(X^{T}\right),
\end{gathered}
$$

(more details can be found in $[15,17,18]$ ).

Since the cobordisms $\overline{\mathcal{B}}$ and $\overline{\mathcal{C}}$ can be decomposed as $\overline{\mathcal{B}}=\mathcal{B} \circ \tau_{-+}$and $\overline{\mathcal{C}}=\tau_{-+} \circ \mathcal{C}$, 

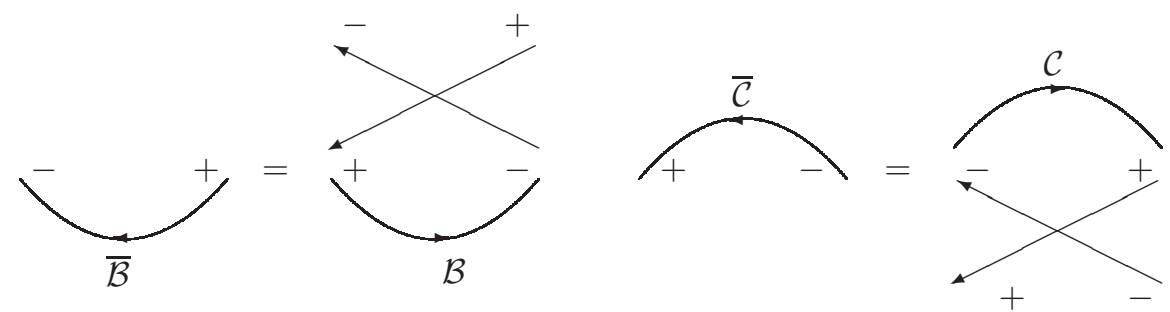

it follows that $F(\overline{\mathcal{B}})=F(\mathcal{B}) \cdot F\left(\tau_{-+}\right)$and $F(\overline{\mathcal{C}})=F\left(\tau_{-+}\right) \cdot F(\mathcal{C})$, i.e.

$$
\begin{aligned}
& F(\overline{\mathcal{B}})=\left[\begin{array}{lll|lll|l|lll}
\beta_{11} & \ldots & \beta_{1 q} & \beta_{21} & \ldots & \beta_{2 q} & \ldots & \beta_{p 1} & \ldots & \beta_{p q}
\end{array}\right] \cdot S_{q p} \\
& \stackrel{(4.3)}{=}\left[\begin{array}{lll|lll|l|lll}
\beta_{11} & \ldots & \beta_{p 1} & \beta_{12} & \ldots & \beta_{p 2} & \ldots & \beta_{1 q} & \ldots & \beta_{p q}
\end{array}\right], \\
& F(\overline{\mathcal{C}})=S_{q p} \cdot\left[\begin{array}{llll|lll|l|lll}
\gamma_{11} & \ldots & \gamma_{1 p} & \gamma_{21} & \ldots & \gamma_{2 p} & \ldots & \gamma_{q 1} & \ldots & \gamma_{q p}
\end{array}\right]^{T}
\end{aligned}
$$

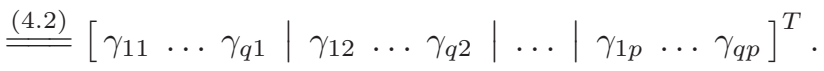

Analogously, we can decompose the cobordism $i d_{-}$into two cobordisms $i d_{-} \otimes \overline{\mathcal{C}}$ and $\overline{\mathcal{B}} \otimes i d_{-}$,

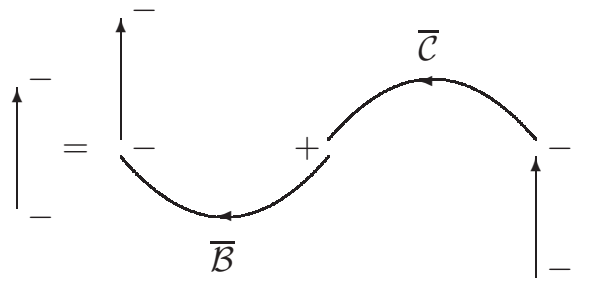

Applying $F$, we see that

$$
\begin{array}{r}
i d_{F(-)}=\left(F(\overline{\mathcal{B}}) \otimes i d_{F(-)}\right) \cdot\left(i d_{F(-)} \otimes F(\overline{\mathcal{C}})\right), \text { i.e. } \\
E_{q}=\left(F(\overline{\mathcal{B}}) \otimes E_{q}\right) \cdot\left(E_{q} \otimes F(\overline{\mathcal{C}})\right),
\end{array}
$$

where $E_{q}$ is the identity matrix of order $q$. This yields the system of $q^{2}$ equations

$$
\sum_{i=1}^{p} \beta_{i k} \cdot \gamma_{l i}=\delta_{k l}, \quad k, l \in\{1, \ldots, q\},
$$

equivalent to the matrix equation

$$
Y \cdot X=E_{q} .
$$

Consequently, the matrix $X \in \mathcal{M}_{p \times q}$ has a left inverse, so its columns are linearly independent, i.e. its column rank is equal to $q$. Since the row rank and the column rank of a matrix are equal, it follows that $p=q$ as claimed. Note that we have proved more, namely that the matrices $X$ and $Y$ are inverses to each other. 
The object part of every strict 1-TQFT is completely determined by the image $p$ of positively oriented point. By the Proposition 4.2 the value of $F$ on any object $a_{1} \ldots a_{n}$ of $1 C o b$, consisting of $k$ positively and $l$ negatively oriented points, is $p^{k} \cdot p^{l}=p^{k+l}=p^{n}$. Hence, every strict 1-TQFT, mapping the manifold consisting of one point to a number $p \geq 2$, on objects coincide with Brauerian representation. Since every cobordism is a finite tensor product of connected cobordisms composed with some applications of $\tau$ 's, we only need to know where $F$ sends $i d_{+}, i d_{-}, \mathcal{B}, \overline{\mathcal{B}}, \mathcal{C}, \overline{\mathcal{C}}$ and $S^{1}$. The matrices $F\left(i d_{+}\right)$ and $F\left(i d_{-}\right)$are equal to the identity matrix $E_{p}$ of order $p$. Due to the fact that the cobordisms $\overline{\mathcal{B}}$ and $\overline{\mathcal{C}}$ can be decomposed into compositions of the cobordism $\tau_{-+}$with $\mathcal{B}$ and $\mathcal{C}$, respectively, and the equality $F\left(\tau_{-+}\right)=S_{p p}$, we only need to describe the following matrices

$$
\begin{aligned}
& F(\mathcal{B})=\left[\begin{array}{lll|lll|l|lll}
\beta_{11} & \ldots & \beta_{1 p} & \beta_{21} & \ldots & \beta_{2 p} & \ldots & \beta_{p 1} & \ldots & \beta_{p p}
\end{array}\right] \in \mathcal{M}_{1 \times p^{2}},
\end{aligned}
$$

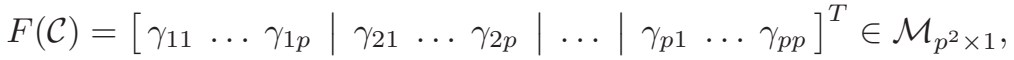

and $F\left(S^{1}\right) \in \mathcal{M}_{1 \times 1}$.

Proposition 4.3. $F\left(S^{1}\right)=p$.

Proof. Let us look at $S^{1}$ as the composition $\mathcal{B} \circ \tau_{-,+} \circ \mathcal{C}$.

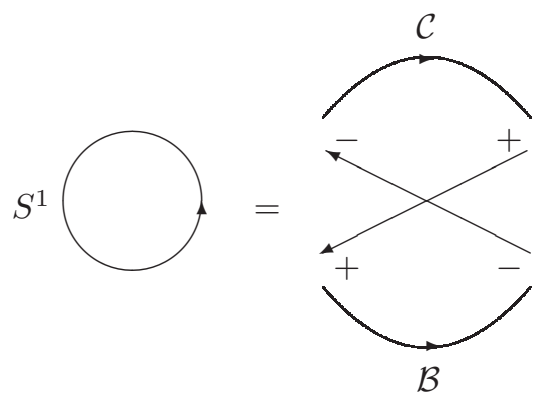

Thus we see that $F\left(S^{1}\right)=F(\mathcal{B}) \cdot F\left(\tau_{-+}\right) \cdot F(\mathcal{C})$, i.e.

$$
\begin{aligned}
& F\left(S^{1}\right)= {\left[\beta_{11} \ldots \beta_{1 p}\left|\beta_{21} \ldots \beta_{2 p}\right| \ldots \mid \beta_{p 1} \ldots \beta_{p p}\right] \cdot S_{p p} } \\
& \cdot\left[\gamma_{11} \ldots \gamma_{1 p}\left|\gamma_{21} \ldots \gamma_{2 p}\right| \ldots \mid \gamma_{p 1} \ldots \gamma_{p p}\right]^{T} \\
& \stackrel{(4.2)}{\underline{ }}\left[\beta_{11} \ldots \beta_{1 p}\left|\beta_{21} \ldots \beta_{2 p}\right| \ldots \mid \beta_{p 1} \ldots \beta_{p p}\right] \\
& \\
& \cdot\left[\gamma_{11} \ldots \gamma_{p 1}\left|\gamma_{12} \ldots \gamma_{p 2}\right| \ldots \mid \gamma_{1 p} \ldots \gamma_{p p}\right]^{T} \\
&= \underbrace{\beta_{11} \gamma_{11}+\beta_{12} \gamma_{21}+\ldots+\beta_{1 p} \gamma_{p 1}}_{1}+\underbrace{\beta_{21} \gamma_{12}+\beta_{22} \gamma_{22}+\ldots+\beta_{2 p} \gamma_{p 2}}_{1} \\
&+\cdots+\underbrace{\beta_{p 1} \gamma_{1 p}+\beta_{p 2} \gamma_{2 p}+\ldots+\beta_{p p} \gamma_{p p}}_{1} \stackrel{(4.1)}{=} p .
\end{aligned}
$$


We can rewrite the matrix equation $X \cdot Y=E_{p}$ (see for instance [10, Section 2.8], [18]) as $H(X) \cdot\left(E_{p} \otimes Y\right)=H\left(E_{p}\right)$, i.e.

$$
\begin{aligned}
& {\left[\begin{array}{lll|lll|l|l}
\beta_{11} \ldots & \beta_{1 p} & \beta_{21} & \ldots & \beta_{2 p} & \ldots & \beta_{p 1} \ldots \beta_{p p}
\end{array}\right] \cdot\left(E_{p} \otimes Y\right)} \\
& =\left[\begin{array}{llll|llll|l|llll}
1 & 0 & \ldots & 0 & 0 & 1 & \ldots & 0 & \ldots & 0 & 0 & \ldots & 1
\end{array}\right] \text {, }
\end{aligned}
$$

as well as $\left(X \otimes E_{p}\right) \cdot L(Y)=L\left(E_{p}\right)$, i.e.

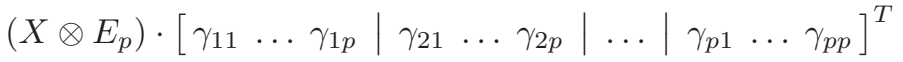

$$
\begin{aligned}
& =\left[\begin{array}{llll|llll|l|llll}
1 & 0 & \ldots & 0 & 0 & 1 & \ldots & 0 & \ldots & 0 & 0 & \ldots & 1
\end{array}\right]^{T} .
\end{aligned}
$$

Since the Brauerian representation assigns the matrices

$$
\left[\begin{array}{lllllllll|l|llll}
1 & 0 & \ldots & 0 & 0 & 0 & 1 & \ldots & 0 & \ldots & 0 & 0 & \ldots & 1
\end{array}\right]
$$

and

$$
\left[\begin{array}{lllllllll|l|llll}
1 & 0 & \ldots & 0 & & 0 & 1 & \ldots & 0 & \ldots & 0 & 0 & \ldots & 1
\end{array}\right]^{T}
$$

to the cobordisms $\mathcal{B}$ and $\mathcal{C}$, respectively, we conclude that every strict 1-TQFT on $\mathcal{B}$ and $\mathcal{C}$ coincides with the Brauerian representation up to multiplication by invertible matrices.

Proposition 4.4. Let $F: 1 C o b \rightarrow M a t_{\mathbb{K}}$ be a strict $1-T Q F T$ such that $F(+)=p \geq 2$, and $B: 1 C o b \rightarrow M a t_{\mathbb{K}}$ be the Brauerian representation. Then, there is a monoidal natural isomorphism $\theta: B \Rightarrow F$.

Proof. Let us assign to each object $a$ of $1 C o b$ an invertible morphism $\theta_{a}: B(a) \rightarrow F(a)$ of $M a t_{\mathbb{K}}$, i.e. an invertible matrix in the following way. We first define $\theta_{\emptyset}: 1 \rightarrow 1, \theta_{+}: p \rightarrow p$ and $\theta_{-}: p \rightarrow p$ to be $E_{1}, E_{p}$ and $X^{-1}$, respectively. Then, for every object $a=a_{1} \ldots a_{n}$ of $1 C o b$ we define $\theta_{a}$ by the Kronecker product $\theta_{a_{1}} \otimes \ldots \otimes \theta_{a_{n}}$. We proceed to show that for every morphism $f: a \rightarrow a^{\prime}$ of $1 C o b$ the following diagram commutes in $M a t_{\mathbb{K}}$.

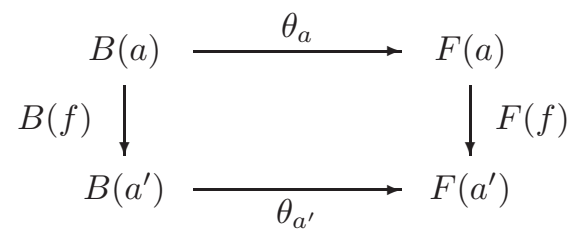

It suffices to prove for generators $i d_{+}, i d_{-}, S^{1}, \mathcal{B}, \mathcal{C}, \overline{\mathcal{B}}$ and $\overline{\mathcal{C}}$. From $B\left(i d_{+}\right)=F\left(i d_{+}\right)=E_{p}$ and $B\left(i d_{-}\right)=F\left(i d_{-}\right)=E_{p}$, we obtain $\theta_{+} \cdot E_{p}=$ $E_{p} \cdot \theta_{+}$and $\theta_{-} \cdot E_{p}=E_{p} \cdot \theta_{-}$. We have $\theta_{\emptyset} \cdot B\left(S^{1}\right)=F\left(S^{1}\right) \cdot \theta_{\emptyset}$ because $1 \cdot p=p \cdot 1$. The following diagrams commute 


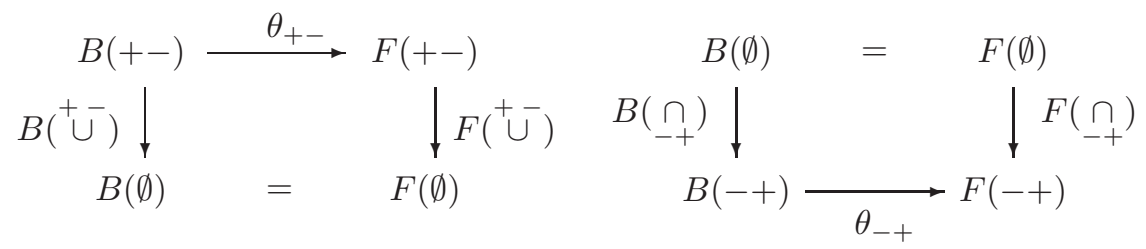

which follows from

$$
B\left({ }^{+} \cup^{-}\right) \stackrel{(4.4)}{=} F\left({ }^{+} \cup^{-}\right) \cdot\left(E_{p} \otimes X^{-1}\right)=F\left({ }^{+} \cup^{-}\right) \cdot\left(\theta_{+} \otimes \theta_{-}\right)=F\left({ }^{+} \cup^{-}\right) \cdot \theta_{+-}
$$

and

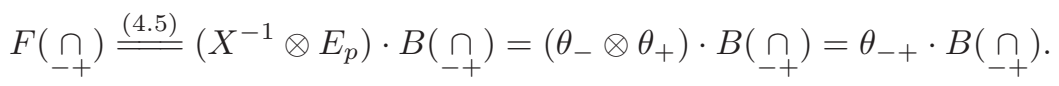

Using the key property of the commutation matrix that enables us to interchange the two matrices of a Kronecker product, we can see that

$$
\begin{aligned}
B\left(\left(^{+}\right)\right. & =B\left({ }^{+} \cup^{-}\right) \cdot S_{p p}=F\left({ }^{+} \cup^{-}\right) \cdot\left(E_{p} \otimes X^{-1}\right) \cdot S_{p p} \\
& =F\left({ }^{+} \cup^{-}\right) \cdot S_{p p} \cdot\left(X^{-1} \otimes E_{p}\right)=F\left(\stackrel{\cup}{+}^{+}\right) \cdot\left(X^{-1} \otimes E_{p}\right)=F\left(\left(^{+}\right) \cdot \theta_{-+},\right. \\
F(\underset{+-}{\cap}) & =S_{p p} \cdot F\left({ }_{-+}^{\cap}\right)=S_{p p} \cdot\left(X^{-1} \otimes E_{p}\right) \cdot B\left({ }_{-+}^{\cap}\right) \\
& =\left(E_{p} \otimes X^{-1}\right) \cdot S_{p p} \cdot B\left({ }_{-+}^{\cap}\right)=\theta_{+-} \cdot B(\underset{+-}{\cap}) .
\end{aligned}
$$

A direct consequence of this last result and the faithfulness of the Brauerian representation is as follows.

COROLlary 4.5. Every strict 1-TQFT, F: $1 \mathrm{Cob} \rightarrow \mathrm{Mat}_{\mathbb{K}}$, such that $F(+)=p \geq 2$, is faithful.

\section{Strong 1-dimensional Topological Quantum Field Theories}

The category $V e c t_{\mathbb{K}}$ of finite dimensional vector spaces over a fixed field $\mathbb{K}$ with ordinary tensor product $\otimes$ and 1-dimensional vector space $\mathbb{K}$ as the unit is a symmetric monoidal, but not a strict monoidal. By the universal property of the tensor product, there is a unique isomorphism $\alpha_{V, W, U}: V \otimes(W \otimes U) \cong$ $(V \otimes W) \otimes U$, such that $v \otimes(w \otimes u) \mapsto(v \otimes w) \otimes u$. The structural isomorphisms $\lambda_{V}: \mathbb{K} \otimes V \cong V$ and $\rho_{V}: V \otimes \mathbb{K} \cong V$ are given by $a \otimes v \mapsto a v$ and $v \otimes a \mapsto a v$, respectively. The symmetry is brought by $\sigma_{V, W}: V \otimes W \cong W \otimes V$ defined by $v \otimes w \rightarrow w \otimes v$.

DEFINITION 5.1. Strong 1-TQFT is a strong symmetric monoidal functor $\left(F, F_{0}, F_{2}\right)$ between the strict symmetric monoidal category $\left(1 C o b, \otimes, \emptyset, \tau_{n, m}\right)$ and the non-strict symmetric monoidal category $\left(\right.$ Vect $\left.t_{\mathbb{K}}, \otimes, \mathbb{K}, \alpha, \lambda, \rho, \sigma_{V, W}\right)$ (for the notion of strong monoidal functor see [14]). 
For every closed oriented 0 -dimensional manifold, regarded as a sequence of points $a=a_{1} \ldots a_{n}$, where $a_{i} \in\{+,-\}$, the functor $F$ assigns a vector space $F(a)$, and for every oriented 1-cobordism $K$ from $a$ to $b$ it assigns a linear map $F(K): F(a) \rightarrow F(b)$. The components of a natural transformation $F_{2}$ are isomorphisms $F_{2}(a, b): F(a) \otimes F(b) \stackrel{\cong}{\rightrightarrows} F(a b)$, and $F_{0}: \mathbb{K} \stackrel{\cong}{\rightrightarrows} F(\emptyset)$ is also an isomorphism of $V e c t_{\mathbb{K}}$. Together, they must make the diagrams involving the structural maps $\alpha, \lambda, \rho$, and $\sigma$ commute in $V e c t_{\mathbb{K}}$ (see [14]).

Our goal is to prove that every strong 1-TQFT, $F: 1 C o b \rightarrow V^{2} t_{\mathbb{K}}$, mapping $a_{i} \in\{+,-\}$ to a vector space of dimension at least 2 , is faithful. For this purpose, let us consider the category $V e c t B_{\mathbb{K}}$ whose objects are the ordered pairs $(V, e)$, where $V$ is a finite dimensional vector space, and $e$ is a chosen ordered basis for $V$. The morphisms $(V, e) \rightarrow\left(V^{\prime}, e^{\prime}\right)$ are just the usual linear maps $V \rightarrow V^{\prime}$. If $e=\left[e_{0}, \ldots, e_{n-1}\right]$ and $f=\left[f_{0}, \ldots, f_{m-1}\right]$ are ordered bases for $V$ and $W$, respectively, let $e \otimes f$ denote the ordered basis for $V \otimes W$, having vector $\varphi\left(e_{i}, f_{j}\right)$ at the $[i \cdot m+j]$-th position, where $\varphi: V \times W \rightarrow V \otimes W$ is canonical bilinear map. The monoidal structure of $V e c t B_{\mathbb{K}}$ is given on objects by

$$
(V, e) \otimes(W, f)=(V \otimes W, e \otimes f),
$$

with $\left(\mathbb{K}, 1_{\mathbb{K}}\right)$ serving as the unit. The tensor product of two morphisms is defined in the same way as in $V e c t_{\mathbb{K}}$.

We now proceed to introduce a new functor $F^{*}: 1 C o b \rightarrow V e c t B_{\mathbb{K}}$. It is recursively defined on objects in the following way, while it coincides with $F$ on morphisms.

The image of $1_{\mathbb{K}}$ under the isomorphism $F_{0}: \mathbb{K} \cong F(\emptyset)$ is taken to be the basis of $F(\emptyset)$, so we set $F^{*}(\emptyset)=\left(F(\emptyset), F_{0}\left(1_{\mathbb{K}}\right)\right)$.

Once we have chosen bases $e_{+}$and $e_{-}$for the spaces $F(+)$ and $F(-)$, respectively, we define $F^{*}(+)=\left(F(+), e_{+}\right)$and $F^{*}(-)=\left(F(-), e_{-}\right)$. If $a=a_{1} \ldots a_{n}$ is an object of length $n$, we define $F^{*}\left(a_{1} \ldots a_{n}\right)=$ $\left(F\left(a_{1} \ldots a_{n}\right), e_{a_{1} \ldots a_{n}}\right)$, where a basis $e_{a_{1} \ldots a_{n}}$ for the space $F\left(a_{1} \ldots a_{n}\right)$ is obtained by taking the image of an ordered basis of domain under the isomorphism

$$
F_{2}\left(a_{1}, a_{2} \ldots a_{n}\right): F\left(a_{1}\right) \otimes F\left(a_{2} \ldots a_{n}\right) \rightarrow F\left(a_{1} \ldots a_{n}\right) .
$$

More precisely, if $e_{a_{1}}$ is a given basis for $F\left(a_{1}\right)$, and $e_{a_{2} \ldots a_{n}}$ is recursively defined basis for $F\left(a_{2} \ldots a_{n}\right)$, then the basis for the space $F\left(a_{1} \ldots a_{n}\right)$ is taken to be the image of basis $e_{a_{1}} \otimes e_{a_{2} \ldots a_{n}}$ under the isomorphism $F_{2}$.

Our next task is to prove that $F^{*}$ is faithful. To do this, take a dimensional functor $G: V e c t B_{\mathbb{K}} \rightarrow M a t_{\mathbb{K}}$, sending a vector space to its dimension, and a linear map to its matrix with respect to the chosen ordered bases

$$
\begin{gathered}
G(V, e)=\operatorname{dim} V, \\
G\left(L:(V, e) \rightarrow\left(V^{\prime}, e^{\prime}\right)\right)=[L]_{e, e^{\prime}} .
\end{gathered}
$$


Lemma 5.2. Let $e=\left[e_{0}, \ldots, e_{n-1}\right], f=\left[f_{0}, \ldots, f_{m-1}\right]$ and $g=$ $\left[g_{0}, \ldots, g_{k-1}\right]$ be bases for $U, V$ and $W$, respectively. If $\alpha:(U, e) \otimes((V, f) \otimes$ $(W, g)) \rightarrow((U, e) \otimes(V, f)) \otimes(W, g)$ is defined by

$$
\alpha\left(e_{i} \otimes\left(f_{j} \otimes g_{h}\right)\right)=\left(e_{i} \otimes f_{j}\right) \otimes g_{h},
$$

then

$$
G(\alpha)=E_{n \cdot m \cdot k},
$$

where $E_{n \cdot m \cdot k}$ is the identity matrix of order $n \cdot m \cdot k$.

Proof. The vector $e_{i} \otimes\left(f_{j} \otimes g_{h}\right)$ is the $[i \cdot(m k)+j \cdot k+h]$-th element of the basis $e \otimes(f \otimes g)$ for the space $U \otimes(V \otimes W)$, and the vector $\left(e_{i} \otimes f_{j}\right) \otimes g_{h}$ is the $[(i \cdot m+j) \cdot k+h]$-th element of the basis $(e \otimes f) \otimes g$ for the space $(U \otimes V) \otimes W$. The image under $\alpha$ of the $l$-th basis vector $e_{i} \otimes\left(f_{j} \otimes g_{h}\right)$ is the $l$-th basis vector $\left(e_{i} \otimes f_{j}\right) \otimes g_{h}$. Thus, the matrix representation of $\alpha$ with respect to bases $e \otimes(f \otimes g)$ and $(e \otimes f) \otimes g$ is the identity matrix of the appropriate order.

LEMMA 5.3. Given any objects $a$ and $b$ of $1 C o b$, we have

$$
G\left(F_{2}(a, b): F(a) \otimes F(b) \stackrel{\cong}{\longrightarrow} F(a b)\right)=E .
$$

Proof. The proof is by induction on the length of the object $a$. Base case: Let $a_{1} \in\{+,-\}$ be an object of length 1 and let $b=a_{2} \ldots a_{n}$ be an object of an arbitrary length. Fix ordered basis $e_{a_{1}}=\left[e_{0}, \ldots, e_{p-1}\right], p \geq 2$, and $e_{a_{2} \ldots a_{n}}=\left[f_{0}, \ldots, f_{m-1}\right]$ for $F\left(a_{1}\right)$ and $F\left(a_{2} \ldots a_{n}\right)$, respectively. Then the corresponding basis $\left[g_{0}, \ldots, g_{p m-1}\right]$ for $F\left(a_{1}\right) \otimes F\left(a_{2} \ldots a_{n}\right)$ is given by $g_{i \cdot m+j}=e_{i} \otimes f_{j}$. The matrix representation of

$$
F_{2}\left(a_{1}, a_{2} \ldots a_{n}\right): F\left(a_{1}\right) \otimes F\left(a_{2} \ldots a_{n}\right) \stackrel{\cong}{\longrightarrow} F\left(a_{1} \ldots a_{n}\right)
$$

with respect to the bases $\left[g_{0}, \ldots, g_{p m-1}\right]$ and $\left[F_{2}\left(g_{0}\right), \ldots, F_{2}\left(g_{p m-1}\right)\right]$ is the identity matrix of order $p \cdot m$, i.e. $G\left(F_{2}\left(a_{1}, b\right)\right)=E$. Induction step: Suppose that the claim is true for all objects $a$ of length less than $n$, where $n>1$. Take an arbitrary object $a=a_{1} \ldots a_{n}$ of $1 C o b$. By the commutativity of the following diagram

$$
\begin{array}{rr}
F\left(a_{1}\right) \otimes\left(F\left(a_{2} \ldots a_{n}\right) \otimes F(b)\right) \stackrel{\alpha}{\longrightarrow}\left(F\left(a_{1}\right) \otimes F\left(a_{2} \ldots a_{n}\right)\right) \otimes F(b) \\
\mathbf{1} \otimes F_{2}\left(a_{2} \ldots a_{n}, b\right) \downarrow & \downarrow F_{2}\left(a_{1}, a_{2} \ldots a_{n}\right) \otimes \mathbf{1} \\
F\left(a_{1}\right) \otimes F\left(a_{2} \ldots a_{n} b\right) & F\left(a_{1} a_{2} \ldots a_{n}\right) \otimes F(b) \\
F_{2}\left(a_{1}, a_{2} \ldots a_{n} b\right) \downarrow & \downarrow F_{2}\left(a_{1} a_{2} \ldots a_{n}, b\right) \\
F\left(a_{1}\left(a_{2} \ldots a_{n} b\right)\right) & =F\left(\left(a_{1} a_{2} \ldots a_{n}\right) b\right)
\end{array}
$$


we have that

$$
\begin{gathered}
F_{2}\left(a_{1} a_{2} \ldots a_{n}, b\right)= \\
F_{2}\left(a_{1}, a_{2} \ldots a_{n} b\right) \circ\left(\mathbf{1} \otimes F_{2}\left(a_{2} \ldots a_{n}, b\right)\right) \circ \alpha^{-1} \circ\left(F_{2}\left(a_{1}, a_{2} \ldots a_{n}\right) \otimes \mathbf{1}\right)^{-1} .
\end{gathered}
$$

Now, by the induction hypothesis and Lemma 5.2 it follows that

$$
G\left(F_{2}\left(a_{1} a_{2} \ldots a_{n}, b\right)\right)=E .
$$

Lemma 5.4. (see [8, Chapter 11, Proposition 17]) Let $L:(V, e) \rightarrow\left(V^{\prime}, e^{\prime}\right)$ and $H:(W, f) \rightarrow\left(W^{\prime}, f^{\prime}\right)$ be linear maps of finite dimensional vector spaces. Then the Kronecker product of matrices $[L]_{e, e^{\prime}}$ and $[H]_{f, f^{\prime}}$, representing $L$

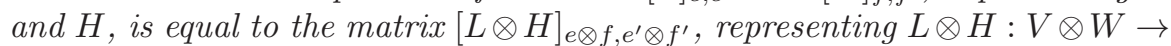
$V^{\prime} \otimes W^{\prime}$, i.e.

$$
G(L \otimes H)=G(L) \otimes G(H) .
$$

Proposition 5.5. The composition $G F^{*}: 1 C o b \rightarrow M_{\mathbb{K}}$ is a strict monoidal functor.

Proof. It can be easily seen that $G F^{*}$ maps unit to unit

$$
\left(G F^{*}\right)(\emptyset)=G\left(F(\emptyset), F_{0}\left(1_{\mathbb{K}}\right)\right)=\operatorname{dim}(F(\emptyset))=1 .
$$

For any two objects $a$ and $b$ of $1 C o b$ we have

$$
\begin{aligned}
\left(G F^{*}\right)(a b) & =G\left(F(a b), e_{a b}\right)=\operatorname{dim} F(a b)=\operatorname{dim}(F(a) \otimes F(b)) \\
& =\operatorname{dim}(F(a)) \cdot \operatorname{dim}(F(b))=G\left(F(a), e_{a}\right) \cdot G\left(F(b), e_{b}\right) \\
& =G\left(F^{*}(a)\right) \cdot G\left(F^{*}(b)\right) .
\end{aligned}
$$

By the naturality of $F_{2}$, the following diagram commutes for every two morphisms $f: a \rightarrow a^{\prime}$ and $g: b \rightarrow b^{\prime}$.

It follows that

$$
\begin{array}{ccc}
F(a) \otimes F(b) & \stackrel{F_{2}(a, b)}{\longrightarrow} & F(a b) \\
F f \otimes F g \downarrow & \downarrow F(f \otimes g) \\
F\left(a^{\prime}\right) \otimes F\left(b^{\prime}\right) & \underset{F_{2}\left(a^{\prime}, b^{\prime}\right)}{\longrightarrow} & F\left(a^{\prime} b^{\prime}\right)
\end{array}
$$

$$
\begin{aligned}
\left(G F^{*}\right)(f \otimes g) & =G(F(f \otimes g))=G\left(F_{2}\left(a^{\prime}, b^{\prime}\right) \circ(F(f) \otimes F(g)) \circ F_{2}^{-1}(a, b)\right) \\
& =G\left(F_{2}\left(a^{\prime}, b^{\prime}\right)\right) \cdot G(F(f) \otimes F(g)) \cdot G\left(F_{2}^{-1}(a, b)\right) \\
& \stackrel{(\text { Lemma 5.3) }}{=} G(F(f) \otimes F(g)) \stackrel{(\text { Lemma 5.4) }}{=} G(F(f)) \otimes G(F(g)) \\
& =\left(G F^{*}\right)(f) \otimes\left(G F^{*}\right)(g) .
\end{aligned}
$$


In our next Proposition we show that the composition $G F^{*}$ maps symmetry to symmetry.

Proposition 5.6. $\left(G F^{*}\right)\left(\tau_{a, b}\right)=S_{n, m}$.

Proof. From the commutativity of the following diagram

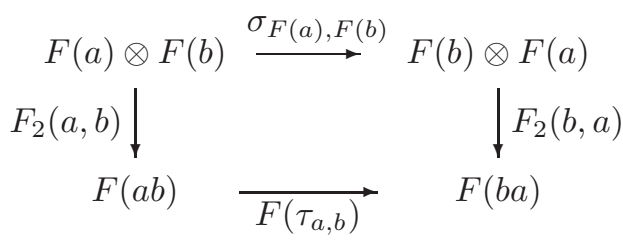

we can see that

$$
\begin{aligned}
& \left(G F^{*}\right)\left(\tau_{a, b}\right)=G\left(F\left(\tau_{a, b}\right)\right)=G\left(F_{2}(b, a) \circ \sigma_{F(a), F(b)} \circ F_{2}^{-1}(a, b)\right) \\
& \quad=G\left(F_{2}(b, a)\right) \cdot G\left(\sigma_{F(a), F(b)}\right) \cdot G\left(F_{2}^{-1}(a, b)\right) \stackrel{(\text { Lemma 5.3) }}{=} G\left(\sigma_{F(a), F(b)}\right) .
\end{aligned}
$$

Since the matrix representation of the linear map $\sigma_{F(a), F(b)}: F(a) \otimes F(b) \rightarrow$ $F(b) \otimes F(a)$ is independent of the choice of the bases $e=\left[e_{0}, \ldots, e_{n-1}\right]$ and $f=\left[f_{0}, \ldots, f_{m-1}\right]$ for $F(a)$ and $F(b)$, we conclude that $G\left(\sigma_{F(a), F(b)}\right)=S_{n, m}$.

Note that we have actually proved that $G F^{*}: 1 C o b \rightarrow M a t_{\mathbb{K}}$ is a strict 1-TQFT. Clearly, $G F^{*}$ satisfies the condition of Corollary 4.5. Therefore, $G F^{*}$ is faithful.

Corollary 5.7. $F^{*}: 1 C o b \rightarrow$ Vect $B_{\mathbb{K}}$ is faithful.

We can now formulate our main result.

THEOREM 5.8. Suppose $\left(F, F_{0}, F_{2}\right): 1 C o b \rightarrow V e c t_{\mathbb{K}}$ is a strong 1 -TQFT, mapping the 0-dimensional manifold consisting of one point to a vector space of dimension at least 2. Then $F$ is faithful.

ACKNOWLEDGEMENTS.

The author is deeply indebted to Professor Zoran Petrić for having kindly suggested the problem and for his inspiring guidance and encouragement throughout the progress of this work, without whose help this paper could not have taken this shape. The author also wishes to thank the anonymous referees for suggestions, which helped to improve the paper. This study was supported by the Ministry of Education, Science, and Technological Development of the Republic of Serbia (Grant 174032).

\section{REFERENCES}

[1] M. Atiyah, Topological quantum field theories, Inst. Hautes Études Sci. Publ. Math. 68 (1988), 175-186. 
[2] Dj. Baralić, Z. Petrić and S. Telebaković, Spheres as Frobenius objects, Theory Appl. Categ. 33 (2018), 691-726.

[3] R. Brauer, On algebras which are connected with semisimple continuous groups, Ann. of Math. 38 (1937), 857-872.

[4] K. Došen and Z. Petrić, A Brauerian representation of split preorders, MLQ Math. Log. Q. 49 (2003), 579-586.

[5] K. Došen and Z. Petrić, Generality of proofs and its Brauerian representation, J. Symbolic Logic 68 (2003), 740-750.

[6] K. Došen and Z. Petrić, Symmetric self-adjunctions: A justification of Brauer's representation of Brauer's algebras, Proceedings of the Conference "Contemporary Geometry and Related Topics" (eds. N. Bokan et al.), Belgrade 2006, 177-187.

[7] K. Došen and Z. Petrić, Symmetric self-adjunctions and matrices, Algebra Colloq. 19 (2012), 1051-1082.

[8] D. Dummit and R. Foote, Abstract algebra, 3rd Edition, John Wiley \& Sons, New York, 2004.

[9] S. Gajović, Z. Petrić and S. Telebaković Onić, A faithful 2-dimensional TQFT, Homology Homotopy Appl. 22 (2020), 391-399.

[10] A. Jain, Fundamentals of digital image processing, Prentice-Hall, Inc., Engelwood Cliffs, New Jersey, 1989

[11] A. Juhász, Defining and classifying TQFTs via surgery, Quantum Topol. 9 (2018), $229-321$.

[12] J. Kock, Frobenius algebras and 2d topological quantum field theories, Cambridge University Press, Cambridge, 2004.

[13] J. Lurie, On the classification of topological field theories, in: Current Developments in Mathematics (eds. D. Jerison et al.), International Press, Somerville 2009, 129-280.

[14] S. Mac Lane, Categories for the working mathematician, second edition, Springer, Berlin, 1998.

[15] J. R. Magnus and H. Neudecker, Matrix differential calculus with applications in statistics and econometrics, 2nd Edition, John Wiley \& Sons, Chichester, 1999.

[16] F. Quinn, Lectures on axiomatic topological quantum field theory, in: Geometry and Quantum Field Theory (eds. D. S. Freed and K. K. Uhlenbeck), Amer. Math. Soc., Providence, 1995, 323-453.

[17] C. Rakotonirina, On the tensor permutation matrices, preprint (arXiv : 1101.0910v3).

[18] W. J. Vetter, Vector structures and solutions of linear matrix equations, Linear Algebra Appl. 10 (1975), 181-188.

[19] E. Witten, Topological quantum field theory, Comm. Math. Phys. 117 (1988), 353386.

S. Telebaković Onić

Faculty of Mathematics

Studentski trg 16, 11000 Belgrade

Serbia

E-mail: sonjat@matf.bg.ac.rs

Received: 7.8.2019. 